\title{
Laterally-resolved mechanical and tribological properties of laser-structured polymer nanocomposites
}

René I. Rodríguez-Beltrán ${ }^{1,2}$, Daniel E. Martínez-Tong ${ }^{3,4,}$, Adela Reyes-Contreras ${ }^{2,5}$, Sandra Paszkiewicz ${ }^{6}$, Anna Szymczyk ${ }^{7}$, Tiberio A. Ezquerra ${ }^{8}$, Pablo Moreno ${ }^{1}$, Esther Rebollar, $^{2 *}$

${ }^{1}$ Grupo de Aplicaciones del Láser y Fotónica (ALF-USAL), Universidad de Salamanca, Pl. de la Merced s/n, 37008 Salamanca, Spain

${ }^{2}$ Instituto de Química Física Rocasolano (IQFR-CSIC), C/Serrano 119, 28006 Madrid, Spain

${ }^{3}$ Donostia International Physics Center (DIPC). P. Manuel Lardizabal 4, Donostia 20018 - Spain

${ }^{4}$ Centro de Física de Materiales (CSIC - EHU/UPV). P. Manuel Lardizabal 5, Donostia 20018 - Spain.

${ }^{5}$ Laboratorio de Investigación y Desarrollo de Materiales Avanzados, Facultad de Química, Universidad Autónoma del Estado de México, Campus Rosedal, Km 14.5 Carretera Toluca-Atlacomulco, San Cayetano de Morelos, Toluca, 50295, Mexico

${ }^{6}$ Institute of Material Science and Engineering, West Pomeranian University of Technology, Szczecin, Piastow Av. 19, PL-70310, Poland

${ }^{7}$ West Pomeranian University of Technology, Institute of Physic, Piastow Av. 48, PL70311

Szczecin, Poland

${ }^{8}$ Instituto de Estructura de la Materia (IEM-CSIC), C/Serrano 121, 28006 Madrid, Spain

*e-mail: danielenrique_martineztong001@ehu.eus, e.rebollar@csic.es

\begin{abstract}
In this work, we report on a detailed quantitative nanomechanical mapping of freestanding films of poly(ethylene terephthalate) (PET) and the composite PET/expanded graphite (EG) with $0.4 \%$ in weight of the nanoadditive, and of these materials nanostructured by laser irradiation. By using atomic force microscopy, we obtained
\end{abstract}


simultaneously the topography, elastic modulus and adhesion force maps of the materials. Young's modulus images exhibited higher values for the composite in comparison to those of the neat polymer and for the nanostructured films in contrast to the non-nanostructured ones. Additionally, we explored the tribological properties of these systems at the nanoscale. Using lateral force microscopy, we observed a decrease in the friction coefficient for the nanocomposite as compared to the neat polymer, while quantifying an increase for both laser-structured samples. Our results are discussed taking into consideration the possible changes that the samples might undergo during processing, as well as the changes imposed by the complex geometry of the nanometric features in these laterally-resolved mechanical measurements.

Keywords. Nanocomposites, laser induced nanostructures, nanomechanical properties. 


\section{Introduction}

Polymer nanocomposites are widely used nowadays in different applications demanding lightweight materials because they offer the possibility to tailor their mechanical properties, especially by controlling the nature of the nanofillers [1-3]. Alumina [4], molybdenum disulfide [5] and carbon-based nanomaterials such as graphite [6], carbon nanotubes [7], and graphene [7] have been previously used to prepare polymer nanocomposites with enhanced mechanical properties. Particularly, polymers reinforced with carbon nanomaterials have shown a significant improvement not only in their mechanical properties [8], but also in their electrical [9] and thermal [10] conductivities, and their barrier impermeability to vapors and gases [11].

A further approach to provide functionality to polymer materials consists in the surface patterning at the micro- and nanoscales $[12,13]$. As an alternative to lithographic methods, which need several step procedures and the use of clean-room facilities, high vacuum or complex mask fabrication, laser surface patterning techniques such as the formation of Laser Induced Periodic Surface Structures (LIPSS) have demonstrated to provide versatility and reliability, and constitute a potential method to obtain large processed surface areas in polymers [14-19]. During LIPSS formation, the polymer surface is heated and the temperature may overcome either the glass transition temperature $\left(T_{g}\right)$ or the melting temperature $\left(T_{m}\right)$ for amorphous and semicrystalline polymers respectively. This effect may allow a rearrangement of the polymer chains. Subsequently, it may lead to the formation of nanostructures parallel to the polarization of the laser beam, with a period close to the laser wavelength [14].

Previous reports showed that polymer surfaces with LIPSS formed upon irradiation with UV nanosecond laser pulses might present changes in their physical properties. For example, contact angle measurements showed that the nanostructured surfaces become more hydrophilic and their surface free energy increased after irradiation [20-22]. From the mechanical properties point of view, the adhesion force in irradiated samples is also modified in comparison to the non-irradiated counterparts [21, 22]. Regarding the tribological properties, the presence of LIPSS on titanium, steel and titanium nitride surfaces has been found to affect the friction and wear coefficients depending on irradiation conditions [23-25]. This effect was explained considering the topographical surface modulation and the chemical modification of the surface, in particular oxidation, 
taking place upon irradiation. In these studies, results were obtained using a macroscopic approach of sliding tests against a hard ball.

Considering the nanometric heights of LIPSS, it is worth performing laterally-resolved physical measurements to test the properties of the ripples individually and thus the molecular implications of LIPSS formation. Previous experiments by us focused on the electrical and piezoelectrical properties of LIPSS on polymers have shown that laser structuring could lead to changes in the molecular organization of the polymers that ultimately affected the final physical behavior [26, 27]. For instance, LIPSS formed on a conjugated polymer showed a heterogeneous electrical conductivity, i.e., electrical transport was lower in the ridges than in the trenches of the ripples, due to the loss of crystallinity during LIPSS formation [26]. To further evaluate the physical implications of LIPSS formation in polymers and in polymer composites, in this work we report on a detailed quantitative nanomechanical mapping on free-standing films of poly(ethylene terephthalate) (PET) and of PET/ expanded graphite (EG) with $0.4 \%$ in weight of EG, and of these materials nanostructured by laser. By using atomic force microscopy, we obtained the topography, elastic modulus, adhesion force and deformation maps of the materials simultaneously. Additionally, using lateral force microscopy, we estimated the friction coefficient for both the non-irradiated and the laser nanostructured materials. This fully atomic force microscopy study allowed to measure the changes in the mechanical properties in the LIPSS ridges and trenches separately, with a lateral resolution better than $40 \mathrm{~nm}$. Moreover, this approach validates the use of nanomechanical measurements to study complex nanostructured materials with complex morphologies.

\section{Experimental}

Films of PET and EG reinforced PET, (PET/EG) with an EG concentration of 0.4 wt.\% were used for this study. The preparation procedure has been reported elsewhere [28, 29]. PET and its composite were melted at $260^{\circ} \mathrm{C}$ and pressed under 5 bar pressure for 2 minutes and under 10 bar pressure for 2 additional minutes. Films with a thickness of approximately $0.4 \mathrm{~mm}$ were obtained. Samples were irradiated using a linearly polarized laser beam from a Q-Switched Nd:YAG system (Lotis TII LS-2131M), at a 
wavelength corresponding to the fourth harmonic of the fundamental $(266 \mathrm{~nm})$, a pulse duration of $8 \mathrm{~ns}$ and a repetition rate of $10 \mathrm{~Hz}$. Irradiation experiments were performed in ambient air at normal incidence and the parameters used in order to obtain LIPSS were 3000 pulses at a fluence of $8 \mathrm{~mJ} / \mathrm{cm}^{2}$ as reported previously by some of us [22].

The topography and nanomechanical properties of the films were evaluated simultaneously by the PeakForce Quantitative Nanomechanical Mapping (PF-QNM) method, using a Bruker Multimode 8 Atomic Force Microscope, equipped with a Nanoscope V controller. All measurements were performed in air at room temperature, using RTESPA300 probes (Bruker). The cantilever spring constant was calculated by the Sader method [30] and found to be around 22-27 N/m. Tip radius calibration was performed against a polystyrene standard of known elastic modulus and found to be in the range 7-12 $\mathrm{nm}$. In PF-QNM, force-distance curves are collected by nanoindentation of the sample in a point-by-point fashion. In this method, the piezo-scanner oscillates at $2 \mathrm{kHz}$ while the probe remains at rest, enabling a high speed and simultaneous capture of force-distance curves and topographic images [31, 32]. The maximum force (peak force) is controlled at each pixel to obtain force-distance curves which are then used as the feedback signal. Analysis of the force-distance curves was performed by using the Nanoscope Analysis software 1.50 that allowed to extract the height, elastic modulus, adhesion force and deformation, simultaneously. Specifically, the elastic modulus was obtained by application of the Derjaguin-Muller-Toporov (DMT) model [33]:

$$
F-F_{\mathrm{adh}}=\frac{4}{3} E^{*} R^{1 / 2} d^{3 / 2}
$$

where $F$ is the force, $F_{\text {adh }}$ the adhesion force, $R$ the tip radius, and $d$ the deformation, i.e. a parameter related to the tip penetration into the sample. $E^{*}$ is the so-called reduced modulus and it is related to the elastic or Young's modulus of the sample by:

$$
\frac{1}{E^{*}}=\frac{1-v_{\text {sample }}^{2}}{E_{\text {sample }}}+\frac{1-v_{\text {probe }}^{2}}{E_{\text {probe }}}
$$

where $v_{\text {sample }}$ and $v_{\text {probe }}$ are the Poisson's ratios of the sample and probe respectively. If the probe Young's modulus $\left(E_{\text {probe }}\right)$ is considered to be much higher than that of the 
sample ( $\left.E_{\text {sample }}\right)$, the second term in the right side of Eq.(2) can be approximated to zero and the elastic modulus of the sample relates to the reduced modulus measured by PFQNM as:

$$
E_{\text {sample }}=\left(1-v_{\text {sample }}^{2}\right) E^{*}
$$

where $v_{\text {sample }}$ is fixed to a constant value of 0.3 in our work. Considering the thickness of the polymer films used in this work $(\approx 0.4 \mathrm{~mm})$, the mechanical impact of the supporting substrate can be neglected.

Nanoscale tribological properties were studied by Lateral Force Microscopy (LFM) at room conditions using the same AFM equipment previously described. LFM experiments were carried out using contact mode SCM-PIC probes (Bruker), having a normal cantilever spring constant around $0.12-0.27 \mathrm{~N} / \mathrm{m}$, as measured by the thermal tune method. This AFM technique works in contact mode, by scanning the sample at a fixed normal force in a direction perpendicular to the cantilever's axis of symmetry. Specifically, in this work the normal force was varied from zero effective load up to the value when plastic sample deformation by tip interaction was observed. All images were recorded at a constant sliding velocity of $4 \mu \mathrm{m} / \mathrm{s}$. In the LFM experiments performed on LIPSS nanostructured samples, the fast-scan direction was kept perpendicular to the orientation of main axis of the LIPSS ripples.

LFM experiments allow calculating the friction force between tip and sample. As the tip scans the surface, the photodetector signal arising from the lateral bending (LB) of the cantilever is recorded in the two scanning directions of a single line, i.e. trace and retrace. From these data, it is possible to calculate a so-called lateral voltage signal $\left(U_{\text {lat }}\right)$, or friction signal, that can be obtained as the mean difference between the forward and backward scan: $U_{\text {lat }}=1 / 2 *\left(\mathrm{LB}_{\text {trace }}-\mathrm{LB}_{\text {retrace }}\right)$. This procedure was carried out offline and allows to eliminate any influence from a tilt or from the topography of the sample. Finally, the lateral voltage signal can be related to the friction force, or lateral force, as previously reported by Schwarz et al. [34]: 


$$
F_{\text {friction }}=\left(\frac{G w t^{3}}{4 L^{2} h}\right)\left(\frac{D_{\text {ver }}}{m}\right) U_{\text {lat }}
$$

where $G$ is the shear modulus of the cantilever, which in our case can be approximated to $50 \mathrm{GPa}$, and $w, t$ and $L$ are the cantilever width, thickness, and length respectively; $h$ is the tip cone height. All the cantilever and tip geometrical parameters were obtained from the manufacturer. $D_{\text {ver }}$ is the vertical cantilever deflection sensitivity that we obtained by measuring a force curve against a fused silica sample and found to be $101 \pm$ $1 \mathrm{~nm} / \mathrm{V}$. Finally, $m=m_{\text {lat }} / m_{\mathrm{ver}} \approx 0.43$ is the relation between the slopes in the lateral $\left(m_{\text {lat }}\right)$ and vertical $\left(m_{\mathrm{ver}}\right)$ directions of the typical calibration curves of the four-segment photodetector.

\section{Results and discussion}

\subsection{Nanomechanical properties}

Figure 1 shows the PFM-QNM images corresponding to PET and PET/EG surfaces before and after laser nanostructuring, taken at a $75 \mathrm{nN}$ peak force. The non-irradiated samples present a smooth surface with a roughness of $3.9 \pm 0.1$ and $4.7 \pm 0.3 \mathrm{~nm}$ for PET and PET/EG, respectively, as measured from their corresponding AFM height images. Upon irradiation, LIPSS are formed with a periodicity of around $270 \mathrm{~nm}$ and $250 \mathrm{~nm}$ for PET and PET/EG respectively, in agreement with our previous work [22]. The nanomechanical properties measured by PF-QNM, i.e., Young's modulus, adhesion and deformation maps, showed homogeneous distributions for both PET and PET/EG (refer to images and profiles in Figure 1). Table 1 lists the average values of the measured nanomechanical properties.

Specifically, the average Young's modulus of PET was $1.6 \mathrm{GPa}$, slightly lower but comparable to the one reported previously by nanoindentation methods $(2.0-2.5 \mathrm{GPa})$ [35]. On the other hand, the PET/EG nanocomposite's Young's modulus showed a mean value of $3.2 \mathrm{GPa}$, representing a $2 \mathrm{x}$ increase in comparison to the neat matrix. The increase of the Young's modulus in a polymer with the addition of carbon nanofillers has been thoroughly reported in the literature [35-37]. However, observing this increase 
at a relatively low concentration of EG $(0.4 \mathrm{wt} \%)$ indicates the excellent dispersion of the nanoadditive into the PET matrix. Also, it is important to highlight that both height images and mechanical maps do not show a visible presence of EG in the evaluated areas, supporting an homogeneous distribution of the EG. Considering that our PFQNM measurements are performed exclusively on the material surfaces, with only a few nanometers penetration depths, the results show that our implementation of the PFQNM technique is sensitive enough to detect mechanical variations related to the samples volume. Finally, the changes in the Young's modulus are accompanied by corresponding variations in both adhesion force and deformation. Thus, the reinforced nanocomposite showed a lower deformation, indicating its increase in hardness.

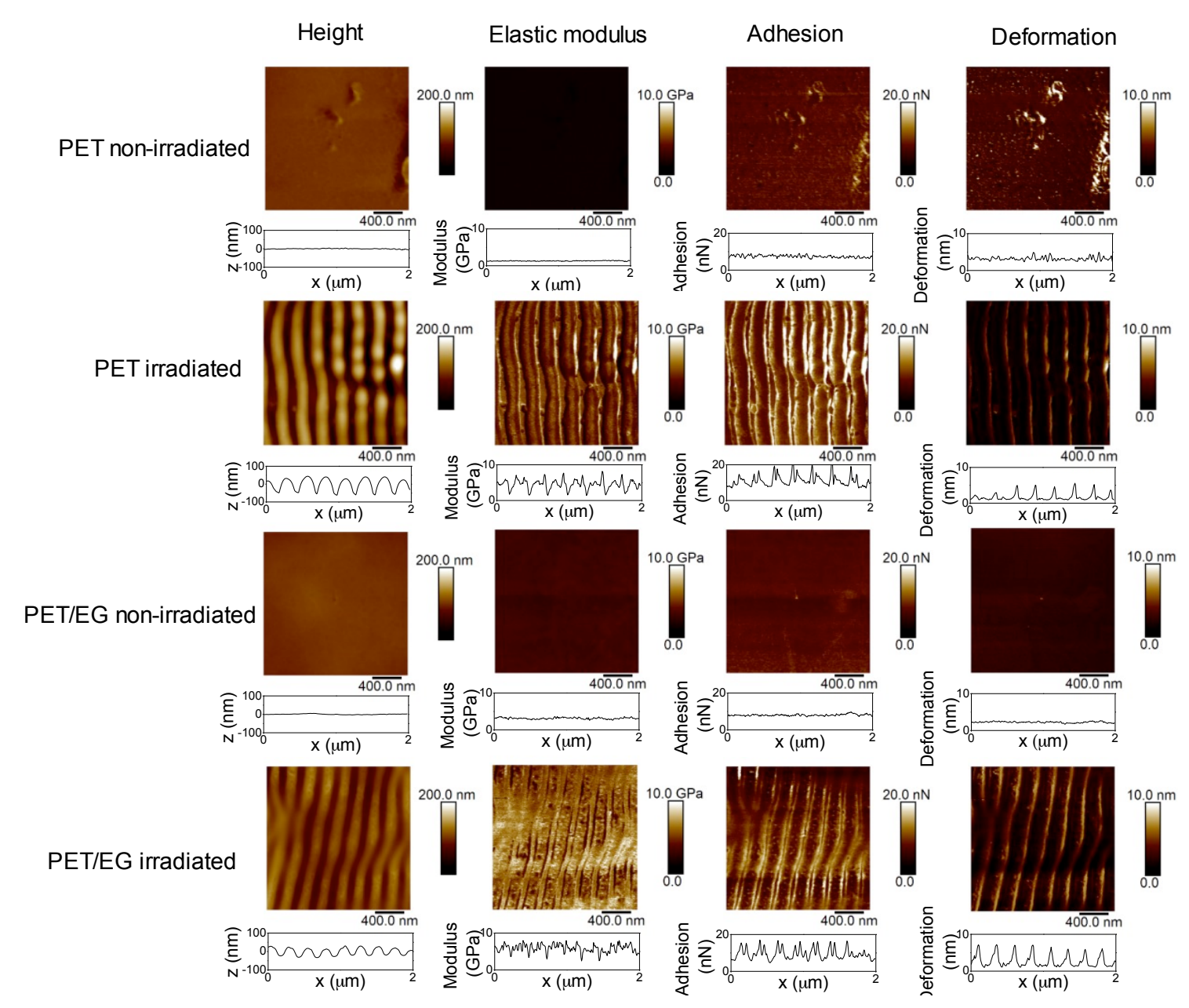

Figure 1. PF-QNM images (height, elastic modulus, adhesion and deformation) of PET and PET/EG non-irradiated and irradiated. 
In contrast to the homogeneous distributions observed in the non-irradiated materials, in both nanostructured films we measured variations in the mechanical properties maps closely related to the topography of the samples. In particular, we quantified different values of Young's modulus, adhesion, and deformation at the slopes of the LIPSS in comparison to those obtained at the peaks and valleys of the nanostructures. To understand these results, we remark that equation (1) (please refer to the Experimental Section) deals with the interaction between a sphere of radius $R$ (tip radius) in contact with a flat and infinite plane (sample surface). Contact between the sphere and the plane takes place in the normal direction. The peaks and valleys of the polymer nanostructures, although not flat, have a radius of curvature much higher than the tip radius, meaning that from the local point of view they can be considered as being "flat enough," and with the tip indenting the sample surface normally. However, the slopes of the LIPSS cannot be modeled appropriately under this approximation, since tipsample contact does not occur under the assumptions of equation (1), and thus the nanomechanical properties values are not reliable. The higher the aspect ratio of the nanostructures, the more serious is the disagreement, as can be seen by the comparison of PET to PET/EG. Since modeling the tip-sample interaction at the slopes of the nanostructures is out of the scope of our current work, we focused on the evaluation of average values corresponding to the top and the valleys of the LIPSS, as summarized in Table 1. We also report that performing measurements scanning parallel and perpendicular to the direction of the ripples did not show differences in the measured nanomechanical properties.

As presented in Table 1, the formation of LIPSS in PET and PET/EG surfaces led to an increase of the Young's modulus and a corresponding decrease in the deformation. PET showed a $2.5 \mathrm{x}$ increase in its Young's modulus, meaning that laser nanostructuring via LIPSS improved the mechanical properties of PET in a comparable way to that observed for the PET/EG raw nanocomposite. This shows the possibility of using LIPSS as a mechanical reinforcing tool for free-standing polymer films, without the need for clean rooms or chemical facilities. In the same line, the PET/EG nanocomposite showed a 2.1x increase in its Young's modulus after LIPSS formation. Adhesion force values measured on the nanostructured samples showed a decrease when compared to the raw films, which might be a consequence of physicochemical modifications induced by laser irradiation, as reported for other polymers [14]. 
Table 1. Nanomechanical mean values for Young's modulus, adhesion force, and deformation for the different samples.

\begin{tabular}{|l|l|l|l|}
\hline & $\begin{array}{l}\text { Young's modulus } \\
(\mathrm{GPa})\end{array}$ & $\begin{array}{l}\text { Adhesion Force } \\
(\mathrm{nN})\end{array}$ & Deformation $(\mathrm{nm})$ \\
\hline PET non-irradiated & $1.6 \pm 0.4$ & $7 \pm 3$ & $3.7 \pm 0.9$ \\
\hline PET irradiated & $4.1 \pm 0.3$ & $2 \pm 1$ & $3 \pm 1$ \\
\hline $\begin{array}{l}\text { PET/EG non- } \\
\text { irradiated }\end{array}$ & $3.2 \pm 0.2$ & $11 \pm 5$ & $2.2 \pm 0.5$ \\
\hline PET/EG irradiated & $6.8 \pm 0.9$ & $9 \pm 1$ & $1.9 \pm 0.7$ \\
\hline
\end{tabular}

\subsection{Nanoscale tribological properties}

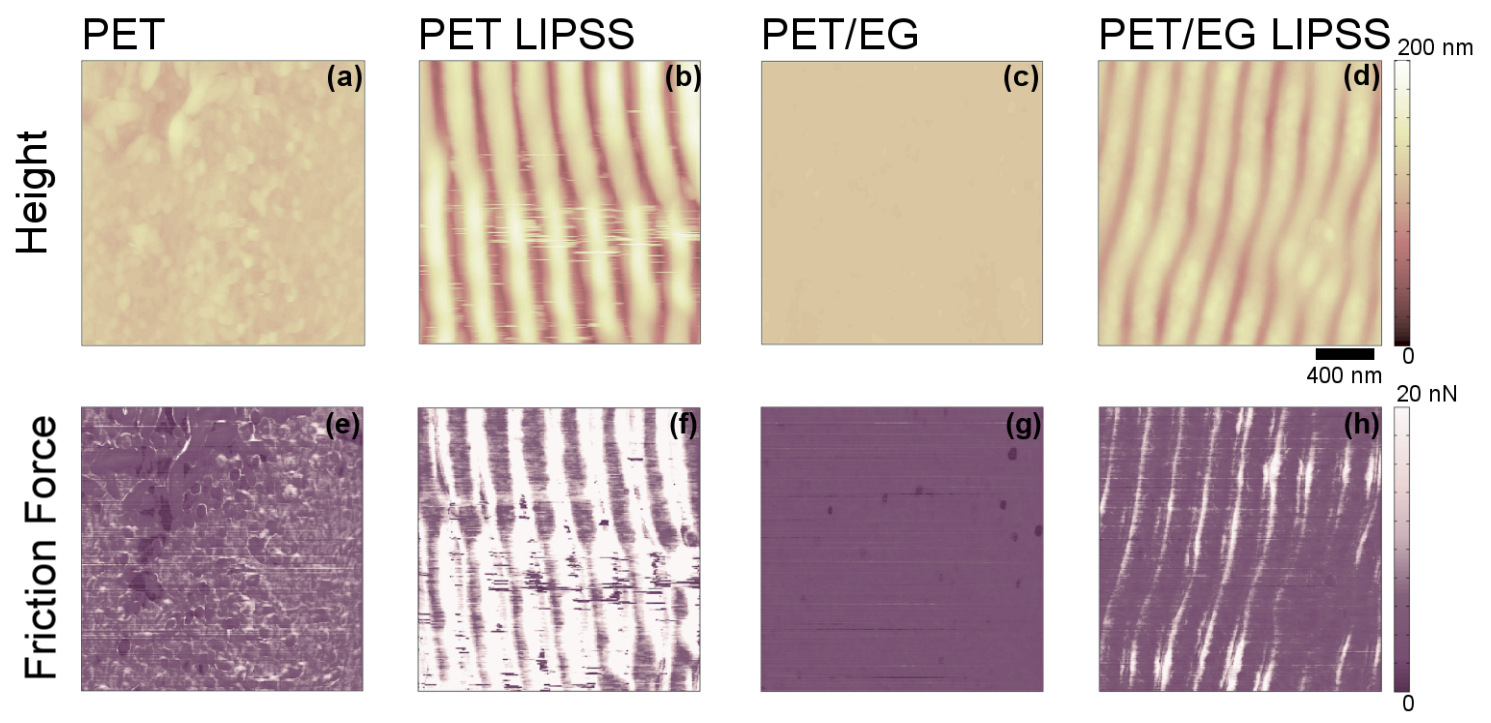

Figure 2. Lateral force microscopy height and friction force maps for non-irradiated and irradiated samples.

Figure 2 shows the height images and friction force maps for all the studied samples, taken at a $4 \mathrm{nN}$ normal force. The height images (top row in Figure 2) show that, in general, the samples do not wear during contact mode scanning; only the nanostructured PET sample (Figure $2 b$ ) shows noisy areas that could be related to enhanced tip-sample interactions. While non-irradiated samples, as well as the irradiated PET/EG ones, could 
stand normal forces of $30 \mathrm{nN}$ and above, the nanostructured PET sample could be only probed up to a $12 \mathrm{nN}$ load. Above this value, surface scratching took place in the nanostructured PET samples. This points out a decrease in the wear resistance for the nanostructured PET surface, in comparison with the other samples, as discussed in the following lines. Notwithstanding, we can state that the use of contact mode AFM for the tribological characterization of laser nanostructured polymers is suitable.

The bottom row of Figure 2 shows the corresponding friction force maps. To provide quantitative data, we performed an offline image processing (i.e., after capture), applying equation (4) in a pixel-by-pixel calculation. During processing, we took into consideration the possible displacement occurring within a unique scan line and corrected it when necessary. For the non-irradiated PET a $4 \mathrm{nN}$ load led to a friction force value of $6 \pm 2 \mathrm{nN}$ while the corresponding value for non-irradiated PET/EG is $4 \pm$ $1 \mathrm{nN}$. These results indicated a small decrease in the friction force for the nanocomposite, at this applied normal force. However, considering the errors in both measurements, it is hard to make a definite conclusion from only single data points. To overcome this problem, we evaluated a wide range of applied normal forces. In Figure 3 we show the mean friction force values as a function of normal force for all the samples.

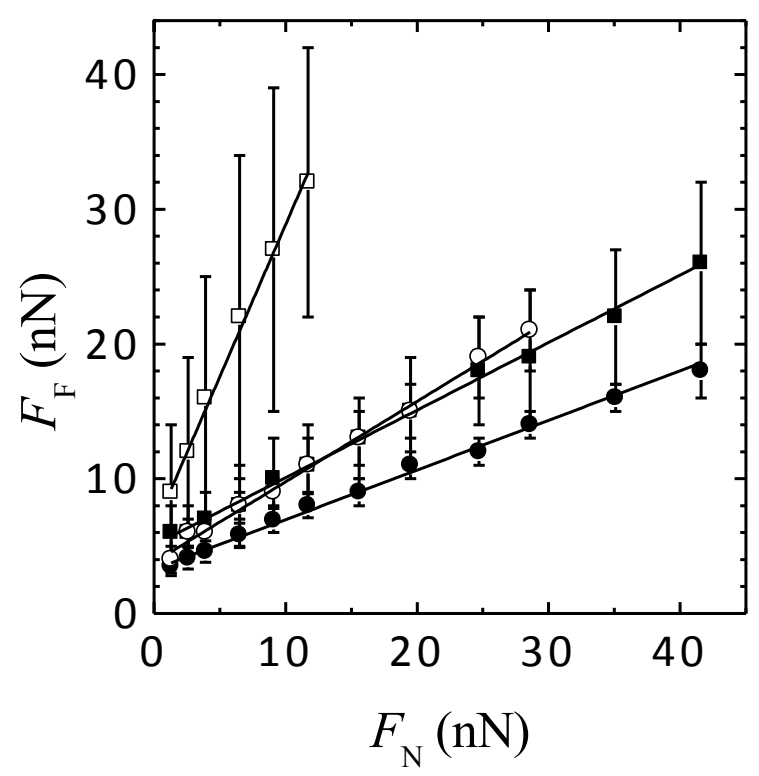

Figure 3. LFM Friction force $\left(F_{\mathrm{F}}\right)$ as a function of the applied normal force $\left(F_{\mathrm{N}}\right)$, for

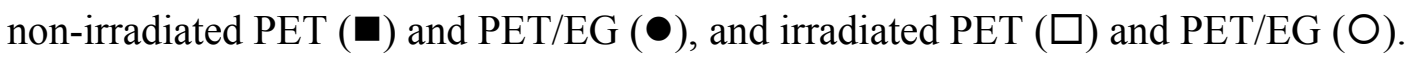


In this figure, filled squares and filled circles represent PET and PET/EG, respectively. The data distribution showed that the reinforced material had smaller friction force values in comparison to the bare polymer matrix. From the quantitative point of view, we analyzed the data in Figure 3 in the framework of the modified version of Amonton's law [38, 39]:

$$
F_{\mathrm{F}}=\mu\left(F_{\mathrm{N}}+F_{0}\right)
$$

where $F_{\mathrm{F}}$ is the measured friction force, $\mu$ the friction coefficient, $F_{0}$ the adhesion force and $F_{\mathrm{N}}$ the applied normal force. The continuous lines in Figure 3 are the fitting of this equation to each data-set. Table 2 summarizes the obtained fitting parameters. From this analysis, we report a friction coefficient $(\mu)$ of about 0.50 for PET and an adhesion force close to $10 \mathrm{nN}$, being this latter value in good quantitative agreement to the one reported by PF-QNM (Table 1). The value of $\mu$ for PET agrees well with the one published by Hurley and Leggett ( $\mu=0.47)$ using friction force microscopy on a bare PET film,[40] although it is slightly higher in comparison to other results in the literature.[41, 42] It is important to recall that PET can crystallize on different conditions and the resulting friction coefficient depends on the polymer crystallinity, as reported by Bhimaraj and collaborators.[41, 42] Also, we carried out these experiments in absence of lubricants as oil, ethanol, or water, and thus a higher friction coefficient is not unexpected.

The PET/EG sample showed lower values of $\mu$ and $F_{0}$ in comparison with the bare matrix; specifically, the friction coefficient decreased by about $25 \%$ while the adhesion force in about $10 \%$. These results claim the role of the expanded graphite as a lubricant in the reinforced PET nanocomposite, at this specific filler concentration and are consistent with the use of graphite as a solid lubricant in polymer-based systems, as discussed in the literature [43-45]. Nonetheless, we cannot extrapolate our results since the variation of $\mu$ and $F_{0}$ in polymer composites does not follow any strict trend, as shown in the literature for several systems [41, 42, 46-48]. For example, investigations in PET-based composites demonstrated that the $\mu$ variation depends not only on the nature of the filler material but also on the filler concentration, giving rise to an increase/decrease or no change in $\mu[41,42]$. 
Table 2. Friction coefficient $(\mu)$ and adhesion force $\left(\mathrm{F}_{0}\right)$ obtained from LFM experiments

\begin{tabular}{|l|l|l|}
\hline Sample & $\mu$ & $F_{0}(\mathrm{nN})$ \\
\hline PET & $0.50 \pm 0.02$ & $10.2 \pm 0.3$ \\
\hline PET EG & $0.37 \pm 0.02$ & $8.9 \pm 0.2$ \\
\hline PET LIPSS & $2.26 \pm 0.07$ & $2.8 \pm 0.3$ \\
\hline PET EG LIPSS & $0.60 \pm 0.02$ & $6.3 \pm 0.3$ \\
\hline
\end{tabular}

Figures 2 (f) and (i) show the friction force maps of the laser nanostructured samples. In both cases, we observed a high contrast among different areas, having the friction values the largest value when probing the slopes of the nanostructures. We measured lower and almost constant friction force values on the LIPSS peaks, like in the PF-QNM analysis. Figure 4 shows height and friction force profiles for comparison.

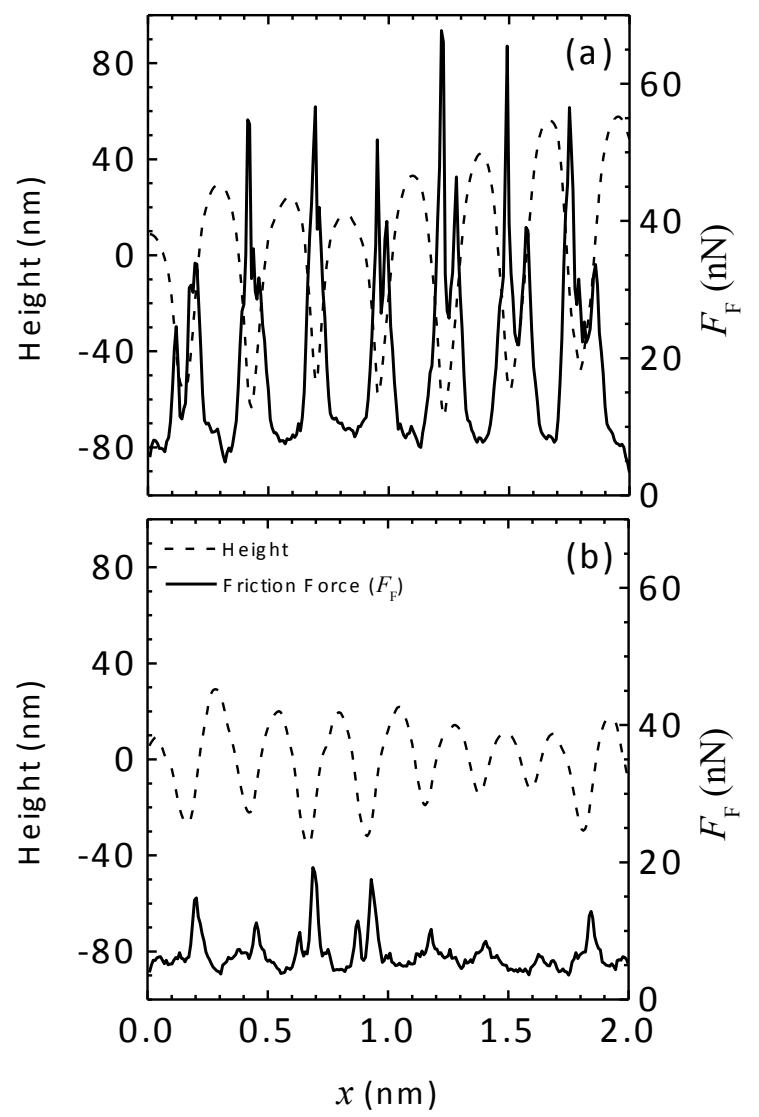

Figure 4. Height and friction force profiles for irradiated PET (a) and irradiated $\mathrm{PET} / \mathrm{EG}(\mathrm{b})$. 
We observe that both samples showed the same general behavior. Friction force changes are more pronounced in the PET sample than in the PET/EG, being the latter less noisy. The friction force maxima quantified in these samples correspond to "residual peaks." These topography-induced effects are not eliminated by the forward and backward scanning protocol, as discussed by Bhushan [49]. Previous reports showed that local variations on the friction force have a stronger dependence on the local surface slope than on the local height distribution [49], as reported in this work. Also, the directionality dependence of the LFM experiments enhances the increase in the friction force, while the collision of the tip against the nanostructures and the possible tip asymmetry might produce additional contributions to the torsion of the cantilever beam, all these factors resulting in a higher friction force [49]. This discussion accounts for the maxima observed for both samples; however, it does not explain why the so-called residual peaks in the nanostructured PET are so intense, in comparison to those for nanostructured PET/EG. Since the LIPSS have a fairly symmetrical geometry, one can calculate the mean slope $(<m>)$ of the nanostructures, going from the bottom of the valleys up to the top of the peaks. We found a $<m>$ PET LIPSS $=0.25$ and $\mathrm{a}<m>_{\mathrm{PET} / \mathrm{EG} \text { LIPSS }}=0.18$, indicating a decrease of almost $30 \%$, i.e. the nanostructured PET/EG sample is flatter, as introduced above for the mechanical modulus analysis. Nonetheless, considering the quantitative differences in the friction force measurements on these two samples demands a further contribution of the EG filler itself to explain the smaller values of friction force as well as a better quality of the measurement for nanostructured PET/EG, beyond the topography-induced effect due to the intrinsic geometry of LIPSS

Having this in mind, we only took into account the friction force values measured on the top of the nanostructures. Figure 3 shows the friction force results for nanostructured PET (open squares) and PET/EG (open circles). It is remarkable the large error bars for nanostructured PET, showing the lack of consistency of the measurements for this sample. The linear fits for the nanostructured samples also correspond to the Amonton's law (equation (5)). Table 2 summarizes the obtained parameters. Both nanostructured samples showed a decrease in the adhesion force compared to the non-irradiated ones, being this result coherent with the adhesion force variations measured by PF-QNM. The friction coefficient increased for both irradiated 
samples. Going into detail, PET/EG showed a 1.6x increase with respect to the nonirradiated film, whereas for the PET sample the increase is $4.5 \mathrm{x}$. Even though $\mu$ is not restricted to be in the $[0,1]$ range, the value found for the nanostructured PET is high and it could be related to an abrasive scanning procedure, explaining as well the noise observed in the topography image. Previously Burton and Bhushan reported an increase in the friction coefficient of polymer nanostructures [50]. In this work, authors observed that relative humidity played a key role on the measurements, as also reported by Feiler et al. [51]. In our experiments, we cannot rule out the possible formation of a thin water layer between the nanostructures patterns, which might interact during LFM scanning, as recently proposed as a hypothesis by Rota and collaborators for LFM measurements in nanostructured inorganic surfaces [52]. However, in this latter work, LFM was carried out using probes with a curvature radius higher than the mean size of the nanostructures, not allowing a local characterization of the material and making hard to conclude about the role of water within the nanostructured channels. We make emphasis on the fact that under our current protocol, we were able to report laterally resolved friction force values on LIPSS and to discuss the nanometric effect these laser structures have on the tribological properties.

\section{Conclusions}

We presented a full atomic force microscopy mechanical and tribological study on PET and on PET/EG nanocomposites free-standing films. We obtained laterally-resolved maps of Young's modulus, adhesion force, deformation and friction force by Peak Force Quantitative Nanomechanical Mapping and Lateral Force Microscopy. We quantified the nanomechanical properties of bare PET and of a nanocomposite of PET reinforced with expanded graphite (EG) $(0.4 \mathrm{wt} \%)$, observing a $2 \mathrm{x}$ increase in the Young's modulus in the nanocomposite. Laser induced periodic surface structures formed on PET and PET/EG showed an increase up to 2.6x in the Young's modulus, compared to the non-irradiated samples. These results emphasize the use of LIPSS as a tool to enhance surface mechanical properties in polymer materials. Using Amonton's law, we estimated the friction coefficient from lateral force microscopy measurements. Our results showed a decrease in the friction coefficient in the PET/EG nanocomposite, in comparison to the bare PET matrix, pointing out the role of EG as solid-state 
lubricant. Laser nanostructured samples showed an increase in the friction coefficient consistent with previous reports in the literature. Our fully microscopy-based approach for the study of nanomechanics in polymer films and polymer nanostructures is proposed as a valid technique to study how contributions arising from complex geometrical features affect the overall mechanical properties, even at surface depths higher than the tip/sample deformation.

\section{Acknowledgements}

The authors gratefully acknowledge financial support from the MINECO (MAT201566443-C02-1-R, CTQ2016-75880-P, FIS2017-87970-R) and Junta de Castilla y León (Project SA287P18). R.I.R.-B. is grateful for the International Scholarship number 314197 provided by Consejo Nacional de Ciencia y Tecnología (CONACyT-México) and E.R. thanks MINECO for a Ramón y Cajal contract (RYC-2011-08069).

\section{References}

[1] S. C. Tjong, Structural and mechanical properties of polymer nanocomposites, Materials Science and Engineering: R: Reports 53 (2006) 73-197.

[2] J. N. Coleman, U. Khan, W. J. Blau, Y. K. Gun'ko, Small but strong: A review of the mechanical properties of carbon nanotube-polymer composites, Carbon 44 (2006) 1624-1652.

[3] P. Parandoush, D. Lin, A review on additive manufacturing of polymer-fiber composites, Composite Structures 182 (2017) 36-53.

[4] C. J. Schwartz, S. Bahadur, Studies on the tribological behavior and transfer filmcounterface bond strength for polyphenylene sulfide filled with nanoscale alumina particles, Wear 237 (2000) 261-273.

[5] S. Wan, Y. Li, J. Peng, H. Hu, Q. Cheng, L. Jiang, Synergistic Toughening of Graphene Oxide-Molybdenum Disulfide-Thermoplastic Polyurethane Ternary Artificial Nacre, ACS Nano 9 (2015) 708-714.

[6] R. Sengupta, M. Bhattacharya, S. Bandyopadhyay, A. K. Bhowmick, A review on the mechanical and electrical properties of graphite and modified graphite reinforced polymer composites, Progress in Polymer Science 36 (2011) 638-670.

[7] G. Mittal, V. Dhand, K. Y. Rhee, S.-J. Park, W. R. Lee, A review on carbon nanotubes and graphene as fillers in reinforced polymer nanocomposites, Journal of Industrial and Engineering Chemistry 21 (2015) 11-25.

[8] S. H. Park, P. R. Bandaru, Improved mechanical properties of carbon nanotube/polymer composites through the use of carboxyl-epoxide functional group linkages, Polymer 51 (2010) 5071-5077.

[9] A. Linares, J. C. Canalda, M. E. Cagiao, M. C. García-Gutiérrez, A. Nogales, I. MartínGullón, et al., Broad-Band Electrical Conductivity of High Density Polyethylene Nanocomposites with Carbon Nanoadditives: Multiwall Carbon Nanotubes and Carbon Nanofibers, Macromolecules 41 (2008) 7090-7097. 
[10] Z. Tao, H. Wang, X. Li, Z. Liu, Q. Guo, Expanded graphite/polydimethylsiloxane composites with high thermal conductivity, Journal of Applied Polymer Science 134 (2017) n/an/a.

[11] D. K. Owens, R. C. Wendt, Estimation of the surface free energy of polymers, Journal of Applied Polymer Science 13 (1969) 1741-1747.

[12] S. Kim, U. T. Jung, S.-K. Kim, J.-H. Lee, H. S. Choi, C.-S. Kim, et al., Nanostructured Multifunctional Surface with Antireflective and Antimicrobial Characteristics, ACS Applied Materials \& Interfaces 7 (2015) 326-331.

[13] J. R. C. Smirnov, M. Ito, M. E. Calvo, C. López-López, A. Jiménez-Solano, J. F. GalisteoLópez, et al., Adaptable Ultraviolet Reflecting Polymeric Multilayer Coatings of High Refractive Index Contrast, Advanced Optical Materials 3 (2015) 1633-1639.

[14] E. Rebollar, M. Castillejo, T. A. Ezquerra, Laser induced periodic surface structures on polymer films: From fundamentals to applications, European Polymer Journal 73 (2015) 162174.

[15] S. Pérez, E. Rebollar, M. Oujja, M. Martín, M. Castillejo, Laser-induced periodic surface structuring of biopolymers, Applied Physics A 110 (2013) 683-690.

[16] P. Slepička, O. Neděla, N. Slepičková Kasálková, P. Sajdl, V. Švorčík, Periodic nanostructure induced on PEN surface by $\mathrm{KrF}$ laser irradiation, International Journal of Nanotechnology 14 (2017) 399-409.

[17] E. Rebollar, S. Pérez, J. J. Hernández, I. Martín-Fabiani, D. R. Rueda, T. A. Ezquerra, et al., Assessment and Formation Mechanism of Laser-Induced Periodic Surface Structures on Polymer Spin-Coated Films in Real and Reciprocal Space, Langmuir 27 (2011) 5596-5606.

[18] E. Rebollar, J. R. V. d. Aldana, J. A. Pérez-Hernández, T. A. Ezquerra, P. Moreno, M. Castillejo, Ultraviolet and infrared femtosecond laser induced periodic surface structures on thin polymer films, Applied Physics Letters 100 (2012) 041106.

[19] M. Forster, W. Kautek, N. Faure, E. Audouard, R. Stoian, Periodic nanoscale structures on polyimide surfaces generated by temporally tailored femtosecond laser pulses, Physical Chemistry Chemical Physics 13 (2011) 4155-4158.

[20] E. Rebollar, S. Perez, M. Hernandez, C. Domingo, M. Martin, T. A. Ezquerra, et al., Physicochemical modifications accompanying UV laser induced surface structures on poly(ethylene terephthalate) and their effect on adhesion of mesenchymal cells, Physical Chemistry Chemical Physics 16 (2014) 17551-17559.

[21] R. I. Rodríguez-Beltrán, S. Paszkiewicz, A. Szymczyk, Z. Rosłaniec, A. Nogales, T. A. Ezquerra, et al., Laser induced periodic surface structures on polymer nanocomposites with carbon nanoadditives, Applied Physics A 123 (2017)

[22] R. I. Rodríguez-Beltrán, M. Hernandez, S. Paszkiewicz, A. Szymczyk, Z. Rosłaniec, T. A. Ezquerra, et al., Laser induced periodic surface structures formation by nanosecond laser irradiation of poly (ethylene terephthalate) reinforced with Expanded Graphite, Applied Surface Science 436 (2018) 1193-1199.

[23] J. Bonse, R. Koter, M. Hartelt, D. Spaltmann, S. Pentzien, S. Höhm, et al., Femtosecond laser-induced periodic surface structures on steel and titanium alloy for tribological applications, Applied Physics A 117 (2014) 103-110.

[24] J. Bonse, R. Koter, M. Hartelt, D. Spaltmann, S. Pentzien, S. Höhm, et al., Tribological performance of femtosecond laser-induced periodic surface structures on titanium and a high toughness bearing steel, Applied Surface Science 336 (2015) 21-27.

[25] J. Bonse, S. V. Kirner, R. Koter, S. Pentzien, D. Spaltmann, J. Krüger, Femtosecond laserinduced periodic surface structures on titanium nitride coatings for tribological applications, Applied Surface Science 418 (2017) 572-579.

[26] Á. Rodríguez-Rodríguez, E. Rebollar, M. Soccio, T. A. Ezquerra, D. R. Rueda, J. V. Garcia-Ramos, et al., Laser-Induced Periodic Surface Structures on Conjugated Polymers: Poly(3-hexylthiophene), Macromolecules 48 (2015) 4024-4031.

[27] D. E. Martinez-Tong, A. Rodriguez-Rodriguez, A. Nogales, M. C. Garcia-Gutierrez, F. Perez-Murano, J. Llobet, et al., Laser Fabrication of Polymer Ferroelectric Nanostructures for Nonvolatile Organic Memory Devices, ACS Appl Mater Interfaces 7 (2015) 19611-19618. 
[28] S. Paszkiewicz, A. Szymczyk, Z. Špitalský, M. Soccio, J. Mosnáček, T. A. Ezquerra, et al., Electrical conductivity of poly(ethylene terephthalate)/expanded graphite nanocomposites prepared by in situ polymerization, Journal of Polymer Science Part B: Polymer Physics 50 (2012) 1645-1652.

[29] A. Szymczyk, Z. Roslaniec, M. Zenker, M. C. Garcia-Gutierrez, J. J. Hernandez, D. R. Rueda, et al., Preparation and characterization of nanocomposites based on $\mathrm{COOH}$ functionalized multi-walled carbon nanotubes and on poly(trimethylene terephthalate), Express Polym. Lett. 5 (2011) 977-995.

[30] J. E. Sader, J. W. M. Chon, P. Mulvaney, Calibration of rectangular atomic force microscope cantilevers, Review of Scientific Instruments 70 (1999) 3967-3969.

[31] C. Marius, L. Y. Seth, V. T. Vladimir, Recent advances in micromechanical characterization of polymer, biomaterial, and cell surfaces with atomic force microscopy, Japanese Journal of Applied Physics 54 (2015) 08LA02.

[32] D. E. Martínez-Tong, A. S. Najar, M. Soccio, A. Nogales, N. Bitinis, M. A. LópezManchado, et al., Quantitative mapping of mechanical properties in polylactic acid/natural rubber/organoclay bionanocomposites as revealed by nanoindentation with atomic force microscopy, Composites Science and Technology 104 (2014) 34-39.

[33] B. V. Derjaguin, V. M. Muller, Y. P. Toporov, Effect of contact deformations on the adhesion of particles, Journal of Colloid and Interface Science 53 (1975) 314-326.

[34] U. D. Schwarz, P. Köster, R. Wiesendanger, Quantitative analysis of lateral force microscopy experiments, Review of Scientific Instruments 67 (1996) 2560-2567.

[35] A. M. Díez-Pascual, M. A. Gómez-Fatou, F. Ania, A. Flores, Nanoindentation in polymer nanocomposites, Progress in Materials Science 67 (2015) 1-94.

[36] C. D. Wood, M. J. Palmeri, K. W. Putz, G. Ho, R. Barto, L. Catherine Brinson, Nanoscale structure and local mechanical properties of fiber-reinforced composites containing MWCNTgrafted hybrid glass fibers, Composites Science and Technology 72 (2012) 1705-1710.

[37] M. Tarfaoui, K. Lafdi, I. Beloufa, D. Daloia, A. Muhsan, Effect of Graphene NanoAdditives on the Local Mechanical Behavior of Derived Polymer Nanocomposites, Polymers 10 (2018)

[38] J. Gao, W. D. Luedtke, D. Gourdon, M. Ruths, J. N. Israelachvili, U. Landman, Frictional Forces and Amontons' Law: From the Molecular to the Macroscopic Scale, The Journal of Physical Chemistry B 108 (2004) 3410-3425.

[39] G. J. Leggett, N. J. Brewer, K. S. L. Chong, Friction force microscopy: towards quantitative analysis of molecular organisation with nanometre spatial resolution, Physical Chemistry Chemical Physics 7 (2005) 1107-1120.

[40] C. R. Hurley, G. J. Leggett, Quantitative Investigation of the Photodegradation of Polyethylene Terephthalate Film by Friction Force Microscopy, Contact-Angle Goniometry, and X-ray Photoelectron Spectroscopy, ACS Applied Materials \& Interfaces 1 (2009) 16881697.

[41] P. Bhimaraj, D. L. Burris, J. Action, W. G. Sawyer, C. G. Toney, R. W. Siegel, et al., Effect of matrix morphology on the wear and friction behavior of alumina nanoparticle/poly(ethylene) terephthalate composites, Wear 258 (2005) 1437-1443.

[42] P. Bhimaraj, D. Burris, W. G. Sawyer, C. G. Toney, R. W. Siegel, L. S. Schadler, Tribological investigation of the effects of particle size, loading and crystallinity on poly(ethylene) terephthalate nanocomposites, Wear 264 (2008) 632-637.

[43] H. Unal, A. Mimaroglu, Friction and wear performance of polyamide 6 and graphite and wax polyamide 6 composites under dry sliding conditions, Wear 289 (2012) 132-137.

[44] K. D. Dearn, T. J. Hoskins, D. G. Petrov, S. C. Reynolds, R. Banks, Applications of dry film lubricants for polymer gears, Wear 298 (2013) 99-108.

[45] A. A. Cenna, P. Dastoor, A. Beehag, N. W. Page, Effects of graphite particle addition upon the abrasive wear of polymer surfaces, Journal of Materials Science 36 (2001) 891-900.

[46] A. Abdelbary, 6 - Wear of polymer composites, in Wear of Polymers and Composites (2014) 133-158.

[47] A. Akinci, S. Sen, U. Sen, Friction and wear behavior of zirconium oxide reinforced PMMA composites, Composites Part B: Engineering 56 (2014) 42-47. 
[48] M. Kalin, M. Zalaznik, S. Novak, Wear and friction behaviour of poly-ether-ether-ketone (PEEK) filled with graphene, WS2 and CNT nanoparticles, Wear 332 (2015) 855-862.

[49] B. Bhushan, Nanotribology and nanomechanics, Wear 259 (2005) 1507-1531.

[50] Z. Burton, B. Bhushan, Hydrophobicity, Adhesion, and Friction Properties of Nanopatterned Polymers and Scale Dependence for Micro- and Nanoelectromechanical Systems, Nano Letters 5 (2005) 1607-1613.

[51] A. A. Feiler, J. Stiernstedt, K. Theander, P. Jenkins, M. W. Rutland, Effect of Capillary Condensation on Friction Force and Adhesion, Langmuir 23 (2007) 517-522.

[52] A. Rota, E. Serpini, G. C. Gazzadi, S. Valeri, AFM-based tribological study of nanopatterned surfaces: The influence of contact area instabilities, Journal of Physics Condensed Matter 28 (2016) 